\title{
Small RNAs in angiosperm gametophytes: from epigenetics to gamete development
}

\author{
Gaël Le Trionnaire and David Twell ${ }^{1}$ \\ Department of Biology, University of Leicester, Leicester LE1 7RH, United Kingdom
}

The established role of various small RNA pathways in the epigenetic regulation of gene expression in the dipolid sporophytic generation of flowering plants contrasts sharply with the lack of knowledge of their role in haploid gametophyte generation. Several recent studies now uncover the operation of multiple small RNA pathways in male and female gametophytes and their essential roles in genome integrity, cell specification, and, most recently, sperm cell function, as described in the May 15, 2010, issue of Genes \& Development by Ron and colleagues (pp. 1010-1021).

Epigenetic phenomena that involve control of the chromatin state and post-transcriptional gene regulation by small RNAs have emerged as key mechanisms governing eukaryotic gene expression. Small RNA molecules and their associated regulatory pathways are thus key effectors of transcriptional and post-transcriptional gene silencing mechanisms, and continue to increase in diversity as new mechanisms are identified. Current knowledge of the diversity and functions of small RNAs in flowering plants has been driven by studies of the leafy sporophyte generation, leaving the haploid gametophyte generation relatively unexplored (Grant-Downton 2010). A suite of recent studies now reveals the operation of multiple small RNA pathways in male and female gametophytes, and uncovers their roles in genome integrity and gamete development. These advances have been driven by the combination of cell isolation procedures, high-throughput sequencing technologies, and genetic analysis. Here we present an overview of the variety of small RNA pathways in plants and the extent to which these pathways are known to contribute to the gametophyte generation of the plant life cycle.

\section{Small RNA pathways in plants}

In the plant world, small RNA pathways are divided into two main classes, depending on the type of RNAs from

[Keywords: ARIADNE; single fertilization; embryo; endosperm; small RNA; ubiquitin ligase]

${ }^{1}$ Corresponding author.

E-MAIL twe@le.ac.uk; FAX 44-116-252-3330.

Article is online at http://www.genesdev.org/cgi/doi/10.1101/gad.1936110. which they originate: microRNAs (miRNAs) and siRNAs. miRNAs are produced from loci that generate ssRNAs that form a hairpin structure that is processed by specific enzymes like DICER-LIKE (DCL1) into 20- to 24nucleotide (nt) small RNAs. These are loaded onto an AGO1-RNA-induced silencing complex (RISC) and guided to the target mRNA through base-pairing. The resulting action on target transcripts can then involve either endonucleolytic cleavage or translational inhibition (Brodersen et al. 2008). miRNAs have been shown to play important roles in plant development, regulating phase transition, hormone biosynthesis and signaling, pattern formation, and morphogenesis (for review, see Chen 2009).

In contrast, siRNAs are produced from longer dsRNAs that are processed into 18- to 25-nt small RNAs. Prominent in this category are siRNAs complementary to heterochromatic regions of the genome. Indeed, transcription of loci composed mainly of noncoding RNAs, transposable elements (TEs), or repeated sequences can give rise to so-called "aberrant RNAs" that form dsRNA regions or that lack normal termini. Such aberrant RNAs are copied into dsRNAs and are processed into 21- to 24nt siRNAs. These are associated with complexes that guide DNA and histone methylation to complementary loci so that potentially damaging regions of the genome can be epigenetically or transcriptionally silenced. Other categories of siRNAs are associated with coding regions of genomes, such as trans-acting siRNAs (ta-siRNAs), where the first cleavage event is initiated by a miRNA. The cleaved products are then used as a matrix to produce dsRNAs that are processed into 21-nt siRNAs directing the post-transcriptional regulation of specific target transcripts. For example, small RNAs derived from Tasi-ARF loci are involved in hormonal regulation of leaf patterning, promoting abaxial identity through repression of the auxin response factors ARF3 and ARF4 (Adenot et al. 2006; Fahlgren et al. 2006; Garcia et al. 2006; Hunter et al. 2006).

Another class of siRNAs arise from loci producing cisnatural antisense transcript pairs (cis-NATs) that are a common feature within eukaryotic genomes $19 \%$ of Arabidopsis genes). It is established that the level of expression of the transcripts constituting the pair can be regulated by the action of 21 - to $24-\mathrm{nt}$ natural cisantisense siRNAs (cis-nat-siRNAs) derived from the 
cleavage of the dsRNA formed by the overlap of the two transcripts (Jin et al. 2008). Although a regulatory role of nat-siRNAs has not been demonstrated in animal models, two recent studies reveal their involvement in stress-responsive gene expression in Arabidopsis. In response to a specific stress condition (pathogen presence or high salt), one transcript of each cis-NAT pair is specifically produced, whereas the second is constitutively expressed. The resulting dsRNAs give rise to specific nat-siRNAs down-regulating the constitutive transcript, thereby producing the appropriate response to stress (Borsani et al. 2005; Katiyar-Agarwal et al. 2006). These represent the first examples clearly establishing the regulatory role of this class of siRNAs in eukaryotes.

\section{miRNA identification in the male gametophyte}

The diversity of small RNAs in plant gametophytes has just begun to be investigated, and the male gametophyte (pollen) has been preferentially studied to date. Indeed, pollen constitutes a more accessible model because of its direct release from the anther, whereas the female gametophyte (embryo sac) is embedded in sporophytic tissues. The mature pollen grain is a simple three-celled structure, which develops from a haploid microspore that first divides asymmetrically to produce a vegetative cell and a generative (or male germ) cell, which then divides once to form two sperm cells (Borg and Twell 2010). Major transcriptomic analyses have revealed complete pollen developmental expression profiles (Honys and Twell 2004; Pina et al. 2005), the sperm cell transcriptome (Borges et al. 2008), and transcriptome changes associated with pollen germination and tube growth (Wang et al. 2008; Qin et al. 2009). Together with the recent discovery of several male gametophytic regulators of cell cycle progression and differentiation (Kim et al. 2008; Brownfield et al. 2009a,b; Chen et al. 2009; Gusti et al. 2009), these studies serve as a foundation to decipher the mechanisms regulating male gamete production and delivery.

These data sets also provide a valuable resource with which to explore the presence of the different small RNA pathways during male gametophyte development. The detection of some transcripts coding for key components of miRNA (such as AGO1 or DCL1) and ta-siRNA (especially $R D R 6$ ) pathways provided a first indication of their operation in Arabidopsis pollen (Grant-Downton et al. 2009a). Further investigation confirmed that miRNAs are conventionally processed, since pre-miRNA, pri-miRNA, and mature miRNA could be detected for miR162 (a regulator of DCL1 transcripts). Moreover, cleavage products of the correct size were detected for different miRNA target transcripts, such as AGO1 (targeted by miR168) and $A R F 16$ and $A R F 17$ (miR160), confirming the function of the miRNA pathway in pollen (Fig. 1). Deep sequencing and Exiqon miRCURY LNA arrays have been used to identify more exhaustively miRNAs expressed in Arabidopsis pollen (Chambers and Shuai 2009; GrantDownton et al. 2009b). Approximately 30 known miRNAs were detected, with the most abundant being miR156, suggesting a role in the repression of flowering-related transcripts in pollen. The abundance of miR158 and miR161 further indicates that PPR protein transcripts may need to be suppressed in pollen. Additional bioinformatic analysis of 454 sequencing data identified seven new species of miRNAs, including miR2939, which is highly gametophyte-enriched and targets a gametophytespecific F-box family transcript for cleavage (GrantDownton et al. 2009b). Interestingly, this transcript is also cleaved in another location by miR774. This unusual double miRNA cleavage event suggests stringent posttranscriptional control of this transcript and important undiscovered roles for proteolytic degradation in gametophyte development and function (Kim et al. 2008; Liu et al. 2008; Gusti et al. 2009).

Undoubtedly, the increases in sequencing depth now available will help to uncover the complete set of gametophyte-expressed miRNAs and their overlap with the sporophyte. These initial studies suggest important roles for miRNAs in pollen development. The analysis of specific classes of miRNAs and their targets is now needed to reveal their functional significance in male gametophyte biology.

\section{siRNAs as silencers of TEs in male and female germlines}

In invertebrates and mammals, germline-specific longer small RNAs of 26-31 nt can be detected. These are called piRNAs because of their specific association with Argonaute family PIWI proteins. Their roles are to direct the silencing of transposons and repeated sequences in germ cells, thereby preserving the genomic integrity of the gametes and preventing the transmission of deleterious effects of active elements to the next generation (Kim et al. 2009). Although such longer small RNAs with potential roles in transposon silencing have not been described in plants, a recent study of Arabidopsis pollen small RNAs reported that 21-nt siRNAs targeting specific TEs (Athila) are present in sperm cells (Slotkin et al. 2009). In contrast, certain classes of TEs were shown to be reactivated and to transpose only in the vegetative nucleus, consistent with the down-regulation of the chromatin remodeling enzyme DECREASED DNA METHYLATION (DDM1) and the absence of the corresponding TE-siRNAs in pollen. Slotkin et al. (2009) thus propose a model whereby the vegetative cell acts as a germline "companion" cell to reveal the presence of intact TEs in the genome and to regulate their activity in the germline by transport of siRNAs into the sperm cells (Fig. 1). In a separate study, the loss of DDM1 from the vegetative cell has been linked to the hypermethylation of centromeric repeats and a class of retrotransposon sequences at non-CG sites (Schoft et al. 2009). Schoft et al. (2009) propose that down-regulation of DDM1 leads to demethylation of $\mathrm{H} 3 \mathrm{~K} 27 \mathrm{me} 2$ and disturbed centromeric heterochromatin, allowing siRNA-directed DNA methylation (RdDM) at centromeric repeats in vegetative cell nuclei. This contrasts with the hypomethylation of some TEs in pollen and their strong non-CG methylation in the sperm cells (Slotkin et al. 2009). A more dynamic view of the distribution of siRNAs and DNA methylation during male gametophyte development 


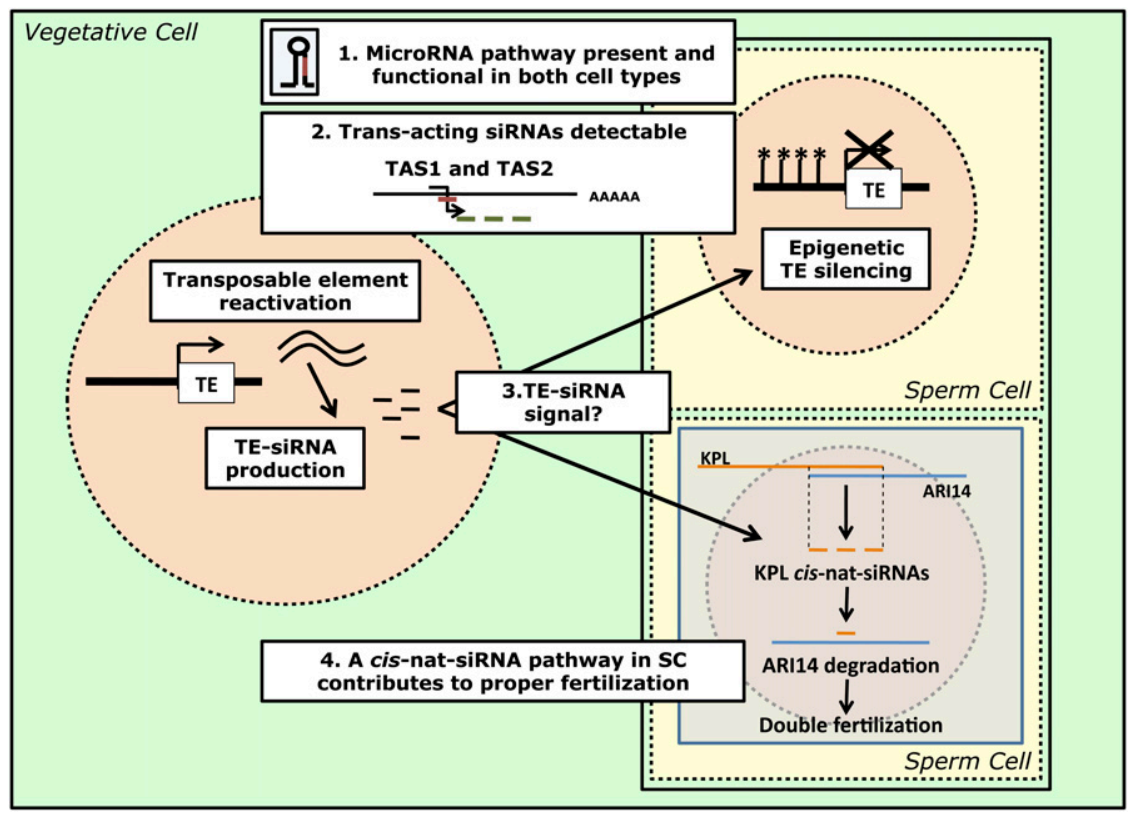

Figure 1. Small RNA pathways in Arabidopsis pollen. In the past few years, several studies have demonstrated the role of different small RNA pathways in the Arabidopsis male gametophyte. (1) Molecular and deep sequencing analyses first demonstrated that miRNAs were detectable and conventionally processed in mature pollen, suggesting the full functionality of the pathway (Grant-Downton et al. 2009a,b). (2) These data also allowed the identification transcripts showing ta-siRNA features. (3) A further study highlights the critical role of siRNA pathways in silencing TEs in germ cells. Slotkin et al. (2009) suggested that the reactivation of TEs in the vegetative cell (associated with the absence of the chromatin remodeling protein DDM1) could serve as a template for the production of TE-siRNAs. Slotkin et al. (2009) propose that these siRNAs constitute a signal that could direct DNA methylation (indicated on the figure by an asterisk $\left[{ }^{\star}\right]$ ) at the complementary loci sperm (DDM1 being present in sperm cells). (4) A recent discovery by Ron et al. (2010) further demonstrates a developmental role for the cis-nat-siRNA pathway in pollen. Indeed, they demonstrate that the KPL/ARI14 NAT pair gives rise to the production of KPL cis-nat-siRNAs, triggering ARI14 degradation. ARI14 control by an siRNA pathway is thought to be a key requirement for proper fertilization, since kpl mutants (unable to give rise to the production of the siRNAs) show failed and "single sperm" fertilization events.

will help to resolve the uncertainties regarding potential siRNA transport and the epigenetic control of different classes of transposon sequences.

An exciting parallel discovery is that the ARGONAUTE 9 (AGO9) protein is necessary to silence TEs in the female gametophyte (Olmedo-Monfil et al. 2010). AGO9 is expressed in sporophytic companion cells surrounding the female gametophyte (but not in the female germ cell lineage) and interacts preferentially with 24-nt siRNAs derived from TEs. Both studies suggest that TEs reactivated in specific accessory cells of both male and female gametophytes enhance siRNA-triggered silencing of TEs in germline cells that is reminiscent of the role of piRNAs in transposon silencing in animals (Mosher and Melnyk 2010). Olmedo-Monfil et al. (2010) also demonstrate that gametophyte precursors are incorrectly specified in ovules of ago9 and several siRNA pathway mutants $(r d r 2, r d r 6$, sgs3, dcl3, and nrpd1a/nrpd1b), resulting in the presence of two female gametophytes in a single ovule. This reveals an important non-cell-autonomous role for a 24-nt siRNA biosynthetic pathway in the restriction of cell fate in the young ovule (Fig. 2). This raises the possibility that some maternal siRNA sequences found in the endosperm (Mosher et al. 2009) and 24-nt siRNAs found in pollen (Slotkin et al. 2009) that resemble AGO9interacting sRNAs may also constitute a similar noncell-autonomous silencing signal (Olmedo-Monfil et al. 2010). The mechanisms involved in the cell-cell transmission of this type of siRNA signal, and whether TE suppression and restriction of cell fate are directly linked, nevertheless remain to be determined.
Regulatory functions for ta-siRNAs and cis-nat-siRNAs in gametophyte development?

Small RNAs and, specifically, siRNAs seem to play a key role in the epigenetic control of gametic development, yet little is known about the putative role of siRNAs in regulating the level of expression of specific transcripts during pollen development. A first indication has been given by Grant-Downton et al. (2009b) in their identification of ta-siRNA features in small RNA populations from Arabidopsis pollen (Fig. 1). Analysis of 454 sequencing data identified phased siRNAs for TAS1 and TAS2 transcripts, suggesting the function of this pathway in mature pollen and a regulatory role for ta-siRNAs in pollen development. In the May 15, 2010, issue of Genes \& Development, Ron et al. (2010) demonstrate that the misregulation of a specific cis-nat-siRNA pair in Arabidopsis sperm cells results in impaired fertilization (Fig. 1). They show that, in wild-type plants, the overlapping transcripts from the KOKOPELLI (KPL)/ARIADNE14 (ARI14) cis-NAT pair exhibit inverse expression patterns during pollen development. This suggested that this pair could give rise to the production of nat-siRNAs originating from KPL transcripts directing down-regulation of ARI14 transcripts in sperm. Analysis of $\mathrm{kpl}$ mutants confirmed the overexpression of ARI14 transcripts in pollen, which was correlated with impaired fertilization involving single fertilization of either the egg or the central cell, leading to seed abortion. Ron et al. (2010) further demonstrated that a KPL/ARI14 nat-siRNA was generated only in sperm cells and was responsible for 




Figure 2. Role of small RNAs in cell fate restriction in the Arabidopsis female gametophyte. Based on comparisons between wild-type and ago 9 mutant phenotypes and the absence of AGO9 in female gametophytes and their precursors, Olmedo-Monfil et al. (2010) suggest that AGO9-associated siRNAs are produced in epidermal cells (L1) of the nucellus through a PolIV-PolVRDR2-DCL3-dependent small RNA pathway. AGO9 complexes were found to associate preferentially with siRNAs derived from TEs, so that siRNA production is proposed to act as a non-cell-autonomous signal (through a RDR6-SGS3-dependent pathway) to restrict cell fate and TE activity. In ovules of ago 9 mutants, a subepidermal nucellar cell gives rise to a supernumerary diploid female gametophyte, and Olmedo-Monfil et al. (2010) suggest that this AGO9-driven siRNA signal normally acts to prevent the specification of a subepidermal cell as a megaspore mother cell (MMC). The MMC normally gives rise after meiosis to a fully functional female gametophyte where TEs are epigenetically silenced, but ago9 mutants show TE reactivation in the egg and synergid cells.
ARI14 down-regulation. KPL encodes a protein of unknown function, while ARI14 is a putative defective ubiquitin E3 ligase. They propose a model for regulation of fertilization by this specific nat-siRNA pair involving a putative role for ARI14 in impairing the function of related ubiquitin E3 ligases (ARI13/ARI15) expressed in sperm cells. Down-regulation of ARI14 by the natsiRNAs would thus prevent the inhibition of ARI13/ ARI15 function, ensuring successful fertilization. Interestingly, the sperm cell-specific KPL gene appears to have evolved recently, as it is present in only some species of Brassicaceae, indicating that the regulation of ARI14 via a KPL/ARI14 nat-siRNA is a novel evolutionary feature. Ron et al. (2010) further highlight the general overexpression of cis-nat-siRNAs in animal testes, and speculate that cis-nat-siRNAs may be a common regulatory mechanism for gene expression in germline cells.

This study provides an intriguing example of a developmental requirement for nat-siRNAs in plant fertilization. It also raises the interesting question of the evolutionary role for these types of NAT pairs and their associated siRNAs. It is conceivable that the gain of KPL through insertion into the $A R I 13 / 14 / 15$ locus may have arisen to suppress mutations in ARI14 (that would normally block specific E3 ligase functions in sperm cells), thereby restoring male fertility. Once established, the essential KPL/ARI14 cis-NAT pair would promote gamete fitness, and the preferential survival of intact cis-NAT genotypes could potentially contribute to population isolation and subspeciation. KPL may also act to promote sperm cell fertility in addition to its role in suppressing ARI14. This issue may be resolved by targeted knockdown of KPL in ari14 mutants (or vice versa), since the juxtaposition of $K P L$ and ARI14 prevents the genetic combination of knockout alleles in germ cells. If loss of ARI14 in a $\mathrm{kpl}$ mutant background fully restores fertility, this would exclude an independent role for KPL in gamete fitness.

\section{Conclusion}

The recent advances uncovering the role of multiple small RNA pathways in the haploid gametophytes of flowering plants are exemplified by the discovery of distinct siRNA pathways controlling genome integrity, cell specification and gamete function. Although this is a promising start, the functions of most small RNAs belonging to miRNA, ta-siRNA, and other cis-nat siRNA categories remain to be explored in the gametophytes. Deep sequencing methods now provide the means for an exhaustive description of the expression profiles of different classes of small RNAs throughout male gametophyte development. The analysis of small RNA diversity in the female gametophyte will be more challenging, but may be overcome through improvements in cellular isolation techniques and sequencing technology. These data sets will undoubtedly lead to new hypotheses of regulatory events underpinning angiosperm gametophyte development to include the contributions of small RNAs to changes in chromatin, DNA methylation, and cell differentiation, and their potential roles in trans-generational epigenetic inheritance.

\section{Acknowledgments}

We are supported by grant funding from the Biotechnology and Biological Sciences Research Council.

\section{References}

Adenot X, Elmayan T, Lauressergues D, Boutet S, Bouche N, Gasciolli V, Vaucheret H. 2006. DRB4-dependent TAS3 trans-acting siRNAs control leaf morphology through AGO7. Curr Biol 16: 927-932.

Borg M, Twell D. 2010. Life after meiosis: Patterning the angiosperm male gametophyte. Biochem Soc Trans 38: $577-582$. 
Borges F, Gomes G, Gardner R, Moreno N, McCormick S, Feijo JA, Becker JD. 2008. Comparative transcriptomics of Arabidopsis sperm cells. Plant Physiol 148: 1168-1181.

Borsani O, Zhu J, Verslues PE, Sunkar R, Zhu JK. 2005. Endogenous siRNAs derived from a pair of natural cisantisense transcripts regulate salt tolerance in Arabidopsis. Cell 123: 1279-1291.

Brodersen P, Sakvarelidze-Achard L, Bruun-Rasmussen M, Dunoyer P, Yamamoto YY, Sieburth L, Voinnet O. 2008. Widespread translational inhibition by plant miRNAs and siRNAs. Science 320: 1185-1190.

Brownfield L, Hafidh S, Borg M, Sidorova A, Mori T, Twell D. 2009a. A plant germ cell-specific integrator of cell cycle progression and sperm specification. PLoS Genet 5: e1000430. doi: 10.1371/journal.pgen.1000430.

Brownfield L, Hafidh S, Durbarry A, Khatab H, Sidorova A, Doerner P, Twell D. 2009b. Arabidopsis DUO POLLEN 3 is a key regulator of male germline development and embryogenesis. Plant Cell 21: 1940-1956.

Chambers C, Shuai B. 2009. Profiling microRNA expression in Arabidopsis pollen using microRNA array and real-time PCR. BMC Plant Biol 9: 87. doi: 10.1186/1471-2229-9-87.

Chen X. 2009. Small RNAs and their roles in plant development. Annu Rev Cell Dev Biol 25: 21-44.

Chen Z, Hafidh S, Poh SH, Twell D, Berger F. 2009. Hyperproliferation caused by loss of Retinoblastoma prevents cell fate establishment during Arabidopsis male gametogenesis. Proc Natl Acad Sci 106: 7257-7262.

Fahlgren N, Montgomery TA, Howell MD, Allen E, Dvorak SK, Alexander AL, Carrington JC. 2006. Regulation of AUXIN RESPONSE FACTOR3 by TAS3 ta-siRNA affects developmental timing and patterning in Arabidopsis. Curr Biol 16: 939-944.

Garcia D, Collier SA, Byrne ME, Martienssen RA. 2006. Specification of leaf polarity in Arabidopsis via the trans-acting siRNA pathway. Curr Biol 16: 933-938.

Grant-Downton RT. 2010. Through a generation darkly: Small RNAs in the gametophyte. Biochem Soc Trans 38: 617-621.

Grant-Downton R, Hafidh S, Twell D, Dickinson HG. 2009a. Small RNA pathways are present and functional in the angiosperm male gametophyte. Molecular Plant 2: 500-512.

Grant-Downton R, Le Trionnaire G, Schmid R, RodriguezEnriquez J, Hafidh S, Mehdi S, Twell D, Dickinson H. 2009b. MicroRNA and tasiRNA diversity in mature pollen of Arabidopsis thaliana. BMC Genomics 10: 643. doi: 10.1186/1471-2164-10-643.

Gusti A, Baumberger N, Nowack M, Pusch S, Eisler $H$, Potuschak T, De Veylder L, Schnittger A, Genschik P. 2009. The Arabidopsis thaliana F-box protein FBL17 is essential for progression through the second mitosis during pollen development. PLoS One 4: e4780. doi: 10.1371/journal.pone. 0004780.

Honys D, Twell D. 2004. Transcriptome analysis of haploid male gametophyte development in Arabidopsis. Genome Biol 5: R85. doi: 10.1186/gb-2004-5-11-r85.

Hunter C, Willmann MR, Wu G, Yoshikawa M, de la Luz Gutierrez-Nava M, Poethig SR. 2006. Trans-acting siRNAmediated repression of ETTIN and ARF4 regulates heteroblasty in Arabidopsis. Development 133: 2973-2981.

Jin H, Vacic V, Girke T, Lonardi S, Zhu JK. 2008. Small RNAs and the regulation of cis-natural antisense transcripts in Arabidopsis. BMC Mol Biol 9: 6. doi: 10.1186/1471-2199-9-6.

Katiyar-Agarwal S, Morgan R, Dahlbeck D, Borsani O, Villegas A, Zhu JK, Staskawicz BJ, Jin H. 2006. A pathogen-inducible endogenous siRNA in plant immunity. Proc Natl Acad Sci 103: $18002-18007$.
Kim HJ, Oh S-A, Brownfield L, Ryu H, Hwang I, Twell D, Nam H-G. 2008. Control of plant male germline proliferation by $\mathrm{SCF}^{\mathrm{FBL} 17}$ degradation of cell cycle inhibitors. Nature 455: 1134-1137.

Kim VN, Han J, Siomi MC. 2009. Biogenesis of small RNAs in animals. Nat Rev Mol Cell Biol 10: 126-139.

Liu J, Zhang Y, Qin G, Tsuge T, Sakaguchi N, Luo G, Sun K, Shi D, Aki S, Zheng N, et al. 2008. Targeted degradation of the cyclin-dependent kinase inhibitor ICK4/KRP6 by RING-type E3 ligases is essential for mitotic cell cycle progression during Arabidopsis gametogenesis. Plant Cell 20: 15381554.

Mosher RA, Melnyk CW. 2010. siRNAs and DNA methylation: Seedy epigenetics. Trends Plant Sci 15: 204-210.

Mosher RA, Melnyk CW, Kelly KA, Dunn RM, Studholme DJ, Baulcombe DC. 2009. Uniparental expression of PolIVdependent siRNAs in developing endosperm of Arabidopsis. Nature 460: 283-286.

Olmedo-Monfil V, Durán-Figueroa N, Arteaga-Vázquez M, Demesa-Arévalo E, Autran D, Grimanelli D, Slotkin RK, Martienssen RA, Vielle-Calzada JP. 2010. Control of female gamete formation by a small RNA pathway in Arabidopsis. Nature 464: 628-632.

Pina C, Pinto F, Feijo JA, Becker JD. 2005. Gene family analysis of the Arabidopsis pollen transcriptome reveals biological implications for cell growth, division control, and gene expression regulation. Plant Physiol 138: 744-756.

Qin Y, Leydon AR, Manziello A, Pandey R, Mount D, Denic S, Vasic B, Johnson MA, Palanivelu R. 2009. Penetration of the stigma and style elicits a novel transcriptome in pollen tubes, pointing to genes critical for growth in a pistil. PLOS Genet 5: 8. doi: 10.1371/journal.pgen.1000621.

Ron M, Alandete Saez M, Eshed Williams L, Fletcher JC, McCormick S. 2010. Proper regulation of a sperm-specific cis-nat-siRNA is essential for double fertilization in Arabidopsis. Genes Dev 24: 1010-1021.

Schoft VK, Chuma N, Mosiole M, Slusarz L, Komnenovic V, Brownfield L, Twell D, Kakutani T, Tamaru H. 2009. Induction of RNA-directed DNA methylation upon decondensation of constitutive heterochromatin. EMBO Rep 10: 1015-1021.

Slotkin RK, Vaughn M, Borges F, Tanurdžić M, Becker JD, Feijó JA, Martienssen RA. 2009. Epigenetic reprogramming and small RNA silencing of transposable elements in pollen. Cell 136: 461-472.

Wang Y, Zhang WZ, Song LF, Zou JJ, Su Z, Wu WH. 2008. Transcriptome analyses show changes in gene expression to accompany pollen germination and tube growth in Arabidopsis. Plant Physiol 148: 1201-1211. 




\section{Small RNAs in angiosperm gametophytes: from epigenetics to gamete development}

Gaël Le Trionnaire and David Twell

Genes Dev. 2010, 24:

Access the most recent version at doi:10.1101/gad.1936110
Related Content Proper regulation of a sperm-specific cis-nat-siRNA is essential for double fertilization in Arabidopsis
Mily Ron, Monica Alandete Saez, Leor Eshed Williams, et al.
Genes Dev. May , 2010 24: 1010-1021
References This article cites 32 articles, 12 of which can be accessed free at:
http://genesdev.cshlp.org/content/24/11/1081.full.html\#ref-list-1
Articles cited in:
http://genesdev.cshlp.org/content/24/11/1081.full.html\#related-urls

\section{License}
Email Alerting
Service
Receive free email alerts when new articles cite this article - sign up in the box at the top right corner of the article or click here.

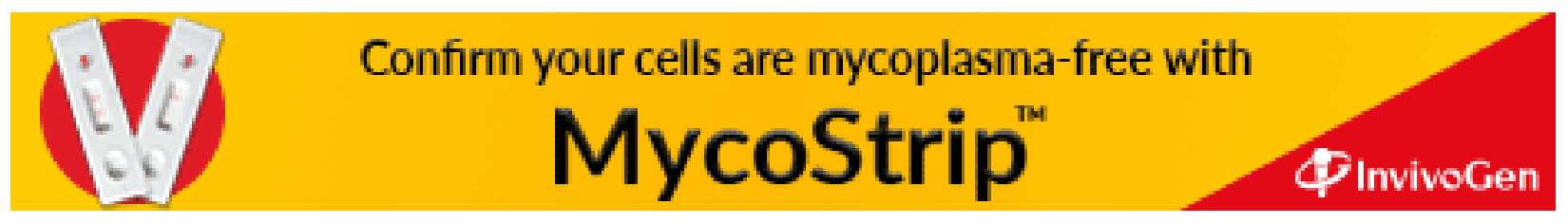

